\title{
Medical ultrasound image segmentation using genetic active contour
}

\author{
Mohammad Talebi, Ahamd Ayatollahi, Ali Kermani
}

Department of Electrical Engineering, Iran University of Science and Technology, Tehran, Iran

Email: Eng.talebi@gmail.com, Ayatollahi@iust.ac.ir, a kermani@elec.iust.ac.ir

Received 10 November 2010; 15 November 2010; 19 November 2010.

\begin{abstract}
Image segmentation is one of the earliest and most important stages of image processing and plays an important role in both qualitative and quantitative analysis of medical ultrasound images but ultrasound images have low level of contrast and are corrupted with strong speckle noise. Due to these effects, segmentation of ultrasound images is very challenging and traditional image segmentation methods may not be leads to satisfactory results. The active contour method has been one of the widely used techniques for image segmentation; however, due to low quality of ultrasound images, it has encountered difficulties. In this paper, we presented a segmental method combined genetic algorithm and active contour with an energy minimization procedure based on genetic algorithms. This method have been proposed to overcome some limits of classical active contours, as contour initialization and local minima (speckle noise), and have been successfully applied on medical ultrasound images. Experimental result on medical ultrasound image show that our presented method only can correctly segment the circular tissue's on ultrasound images.
\end{abstract}

Keywords: Segmentation; Active Contour; Genetic Algorithm; Ultrasound Images

\section{INTRODUCTION}

The ultrasound imaging method is used in medical practices, along with other imaging procedures such as XRay, CT, etc., for producing images of live tissue and for the purpose of clinical diagnosis. Since advantages of ultrasound imaging method such as: being less costly, portability of the device, safety of the imaging technique to the patient, and the less amount of real time required for imaging, it has been paid more attention than other imaging techniques.
Despite all these advantages, ultrasound images, because of characteristic flaws such as speckle noises, and artificial borders, have very low qualities that cause the processing of such images to run into complications and difficulties. Therefore, the implementation of many existing processing algorithms on these images yields no favorable outcomes.

Meanwhile, segmentation is major part of medical image processing. Accurate image segmentation and detection of tissues provides great help to the physicians for clinical diagnosis and tissue classification. So far, many different image segmentation methods have been presented including: the clustering method [1], watershed transform method [2,3], Markov's random field method [4], and methods based on statistical models [5].

The active contour is one of the methods used extensively in recent decades for segmentation of medical ultrasound images [6]. Kass [7] proposed the idea of using active contour for the first time. This method is based on balancing the internal and external forces and minimizing energy. Investigations show that this procedure has produced acceptable results; although, it also has several drawbacks and weak points. The first and the most important drawback is the entrapment of the active contour in the local points of minima, which causes the deviation of the contour from its original path. This problem is magnified in the processing of ultrasound images that have higher noise levels.

To solve this problem, various methods have been suggested, such as the Balloon model [8], the GVF model [9], the distance potential model [10] and etc. More recently, the use of the genetic model besides the active contour has been suggested $[11,12]$. In this paper, we have tried to utilize the genetic algorithm to solve some of active contour problems.

In the section 2, the concept of active contour will be discussed and its weak points delineated. Then, section 3, presents a simple introduction of the genetic algorithm. The proposed method will be explained in section 4 and finally, the obtained results will be evaluated in section 5 . 


\section{INTRODUCING THE ACTIVE CONTOUR}

The idea of using an active contour was first brought up by Kass in 1988, which became known as the snake model [9]. Active contour is a two-dimensional curve in the image space whose deformation is based on energy minimization. In this method, first, a primary contour is defined close to the edge of the object in mind and then, in order to detect the edge, an energy function is specified for contour through various arithmetic techniques, the edge detection and segmentation process is completed. If $x$ and $y$ are the position coordinates of the 2-D image $I(x, y)$, one can use the curve $V(s)=(x(s), y(s))$, in which, $s \in[0,1]$ for parametric representation of the contour on the image. In general, the energy function of the active contour is expressed as:

$$
E_{\text {total }}=\int_{0}^{1} E(V(s)) d s=\int_{0}^{1}\left[E_{\text {int }}(V(s))+E_{\text {ext }}(V(s))\right] d s
$$

The defined energy function for the contour consists of two components which respectively are Internal energy $E_{\text {int }}(V(s))$, External energy $E_{\text {ext }}(V(s))$. The former is used to control the rate of stretch and to prevent discontinuity in the contour the latter is generated by using the image characteristic features or the limitations imposed on the contour by the user, and it is used for contour displacement.

In order to control the contour deformation and displacement, these energy components are converted into two internal and external forces. During the process of contour deformation, the force resulting from internal energy, keeps the contour smooth and prevents breaking and discontinuity of the contour which are caused mainly by the presence of irregularities and noise in the image. Also, the external force has the task of displacing the contour from its initial position and guiding it towards the subject's edge.

The internal energy component can be defined as follow:

$$
E_{\text {int }}(V(s))=\left[\frac{1}{2} \alpha(s)\left|V_{s}(s)\right|^{2}+\frac{1}{2} \beta(s)\left|V_{s s}(s)\right|^{2}\right]
$$

The first term of the internal energy is related to the contour's bending ability. Weight factor $\alpha(s)$ is used to control and adjust it. The second term in this relation specifies the contour's strength and resistance against sudden changes and $\beta(s)$ factor controls it. Equation (3) represents a typical external energy defined for the contour.

$$
E_{\text {ext }}(V(s))=-\gamma\left|\nabla\left[G_{\sigma}(x, y) * I(x, y)\right]\right|^{2}
$$

In this equation, $\gamma$ is the external energy weight factor,
$G_{\sigma}(x, y)$ is a two-dimensional Gaussian function with the standard deviation $\sigma, \nabla$ is the gradient operator and * specifies the convolution operator for the 2-D image $I(x, y)$.

When the energy function attains its minimum value, in other words, when external and internal forces balance out:

$$
F_{\text {int }}+F_{\text {ext }}=0
$$

Contour deformation stops and the edge detection process reaches to the end. This indicates that the contour has coincided with the edge. More details about the energy minimization process have been fully described in reference [9]. The presented model has several deficiencies which are:

- Sensitivity of the contour to defined initial curve position.

- Formation of minimum energy points due to the presence of noise in the image in which entrap contour in these local points and deviate it from the original path.

These weaknesses become more prominent when we try to use the model for the segmentation of ultrasound images. The existence of speckle noise in the image creates many local minima which cause the entrapment of the contour in these points. As was mentioned before, so far, many different methods have been submitted to solve the active contour's problem. Using the genetic algorithm is one way of solving such a problem and obtaining the optimum answer in the search space. In the following section, we will describe the generic algorithm.

\section{GENETIC ALGORITHM}

The genetic algorithm is an efficient, adaptive and stable method of optimization which has caught the attention of the researchers in the last several years. However, these algorithms don't guarantee the optimum solution for problems. Using them for the optimization problems has shown that these algorithms often find the closest solution to the optimum and in some cases; they obtain the most optimum solution among the existing solutions in the search space. The genetic algorithm uses a set of population called chromosomes for optimization. These chromosomes are in fact the solutions of the problem. In a search process, the best of them are selected from the solution set available in the search space. Each of these chromosomes is made up of several genes, and these genes represent the parameters that should be optimized. These chromosomes are encoded by numbers. The manner of encoding these genes and chromosomes depends on the type of problem that needs to be solved. Usually, a string of zeros and ones is used for encoding the chromosomes. After determining the population size and the manner of encoding, the appropriate solution should be 
evaluated. To do this, the fitness function is used. After the fitness function is determined for each member of the population, three genetic operators should be applied on them to prevent premature convergence. These three genetic operators are as follows [13]:

- Selection operator:

This operator is applied on the members of the population and selects a number of individuals who possess the best fitness function values to reproduce the new generation. Different methods exist for the selection of the bests of the society including: selection by the Roulette wheel method, selection by the competition method and the elitist selection method.

- Cross over operator:

After the best members of the society are selected for reproduction, it is necessary for them to cross over and produce the new generation. In this situation, from among selected individuals, certain pairs are identified as parents and by crossing those over two offspring are born. To cross over the parent chromosomes, single point cross over, double point cross over and the uniform cross over methods can be used.

- Mutation operator:

Mutation, by changing the genes, can produce a new chromosome. Applying the mutation operator in the genetic algorithm saves valuable information of the chromosomes which may otherwise be deleted during the execution of the algorithm. In other hand, this act can greatly enhance the algorithm operation and prevent its quick convergence and falling in the trap of local minima. The amount of mutation taking place in a chromosome is determined by the mutation rate. At first, a random number between zero and one is generated for each gene to carry out the mutation operation. If the generated number was smaller than the value of mutation probability, the mutation takes place and the gene changes. Otherwise, no change is carried out on the gene. To calculate the mutation rate, usually, the relation $P_{m}=\frac{1}{L}$ is used, where $L$ is the chromosome length. The process of producing a new generation and selecting the best member is repeated continually until the algorithm's termination condition is satisfied.

\section{GENETIC ACTIVE CONTOUR}

Sensitivity to the initial position of contour and the entrapment within local minima are among the problems inflicted on the active contour model used for image segmentation. This problem is magnified when we try to apply the model to the segmentation of low quality images like an ultrasound images. To resolve this issue, we used the genetic active contour. The process of applying this algorithm has several steps which will be described below.

We first assumed that the tissue under examination is confined within two circles with the radius Rmin and Rmax (Figure 1). In this situation, the specific region under study can be considered as concentric circles, where each of these circles acts like a contour (Figure 2). As the figure shows, each of these contours can contain points from the tissue's edge. So, if we are able to separate these points from each contour and connect them together, we can reveal the tissue's edge. As a first step, we consider a population of chromosomes as the initial population to generate these circles. We then include two genes for every chromosome. The first gene specifies the circle's radius and the second one, the angle of the starting point (Figure 3). After the contours are produced, we mark a number of equidistant points on every on. If $\mathrm{N}$ denotes the number of points on the contour, the distance of every point from the adjacent one would be equal to $\frac{360}{N}$.

Once the initial contours are formed and the contour points are determined, each of these points should be evaluated and the best ones be selected. To do this, first we should define a fitness function. Since the active

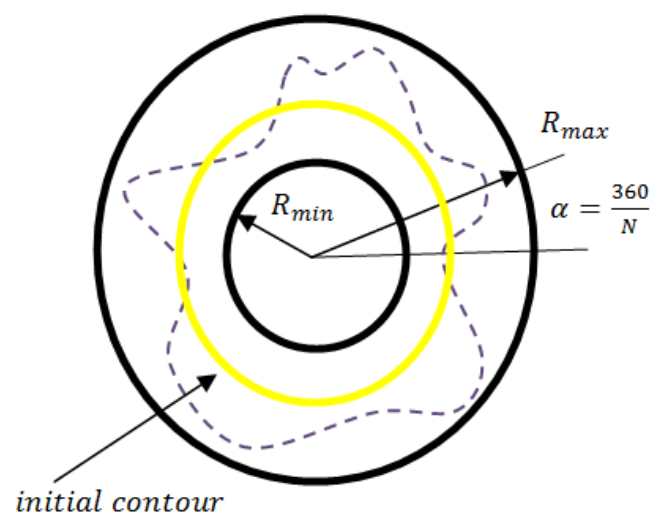

Figure 1. Space where the tissue is confined within two circles.

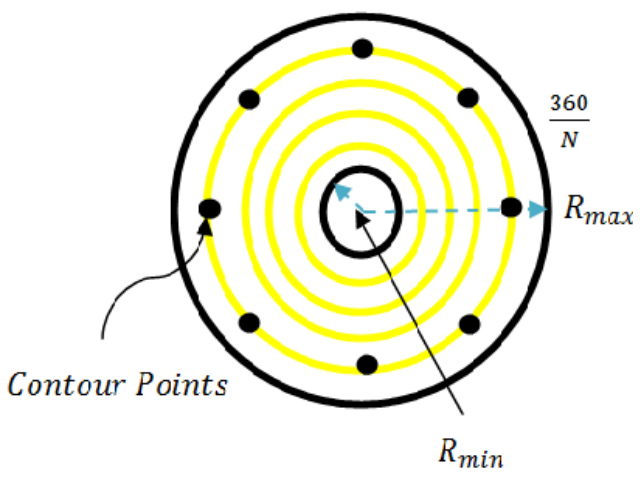

Figure 2. How the initial contours is positioned and the points specified for each contour. 


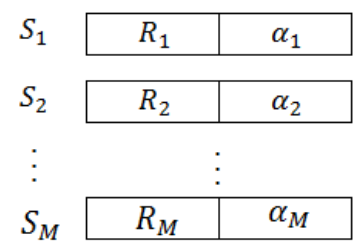

Figure 3. The structure of chromosomes and the production of initial chromosomes. $R M$ represents radius of contours and $\alpha$ specifies the start angle.

contours are founded on the energy minimization principle, therefore the fitness function can be defined as:

$$
\begin{aligned}
& F_{\text {fitness }}=\frac{1}{1+\left(E_{\text {internal }}+E_{\text {external }}\right)}= \\
& \frac{1}{1+\left(\alpha E_{\text {Continuity }}+\beta E_{\text {Curvaure }}+\gamma E_{\text {image }}\right)}
\end{aligned}
$$

With respect to the defined function, by calculating the energy of each contour point, its relevant fitness function can be obtained. Those contour points which are situated on the tissue's edge or in the local minima possess the least amount of energy. So, their fitness functions will have the highest values. Therefore, we calculate the fitness function for the overall contour points to remedy the problem of local minima and to have a better evaluation of all the points at every step, instead of using each single point's fitness function. The overall fitness function for every contour is obtained from the sum of fitness functions of individual contour points. After calculating the overall fitness functions, we select the contours that have the highest values of fitness functions as the best solutions.

At this stage, considering the rate of selection, some of the best individuals from the society are selected and put into the reproduction pool. Then, the members of the pool are paired up and allowed to reproduce. We used the uniform cross over method. Next, to prevent quick convergence and to have a better generation, mutation operation is performed on the chromosome populations. This process is repeated until a set of best contours is finally acquired. After the algorithm runs its course, some of the best individuals are separated and the best points of each contour are selected from the generation that remains. The final contour is obtained by connecting those points.

\section{EXPERIMENTAL RESULTS}

To evaluate the proposed algorithm, it was first applied on a simple image like that of Figure 4-a. As is observed, this image consists of two sections of background and tissue, with some added speckle noise. In this test, 40 points are considered for each contour and the contour energy coefficients are: $\alpha=0.2, \beta=0.35, \gamma=0.13$. This test is repeated for 10,000 times and 100 chromosomes. Figure 4-b illustrates the final result of image segmentation. Figure 5-b shows segmentation result of ultrasound breast lesion (Figure 5-a) for 40 contour points and 10,000 iteration and 40 chromosomes. Energy coefficients are equal to before examination. Figure 6 shows another medical ultrasound image segmentation results by proposed algorithm. Due to initial contours have circular structure, experimental results show that using the mentioned algorithm for the segmentation of circular tissue's as breast tumor in ultrasound images

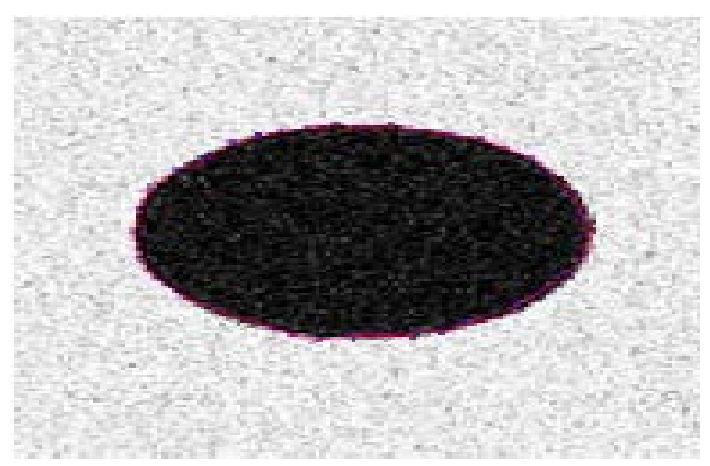

(a)

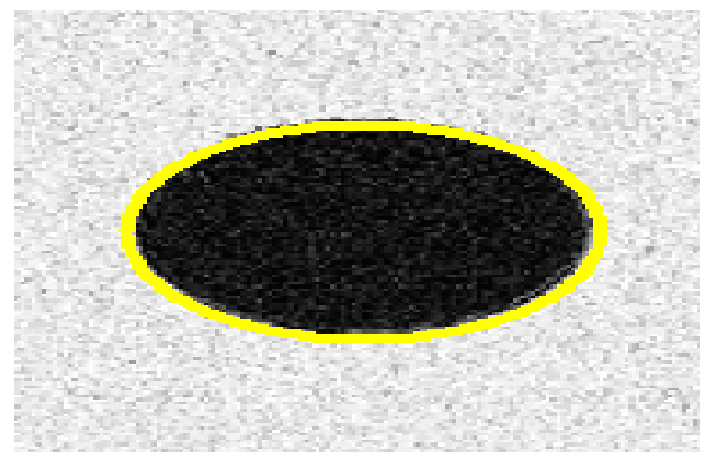

(b)

Figure 4. (a) Original image. (b) Segmented image by means of proposed method

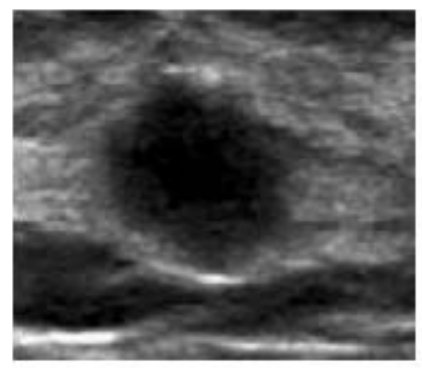

(a)

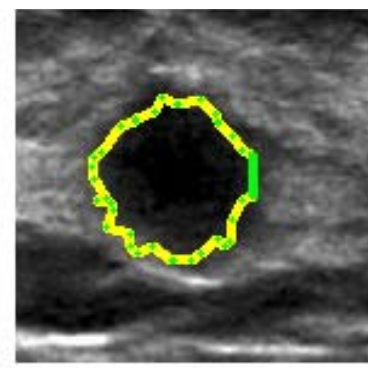

(b)
Figure 5. (a) Anatomical structure from an ultrasound breast lesion. (b) Final result of segmentation after the proposed algorithm is applied. 

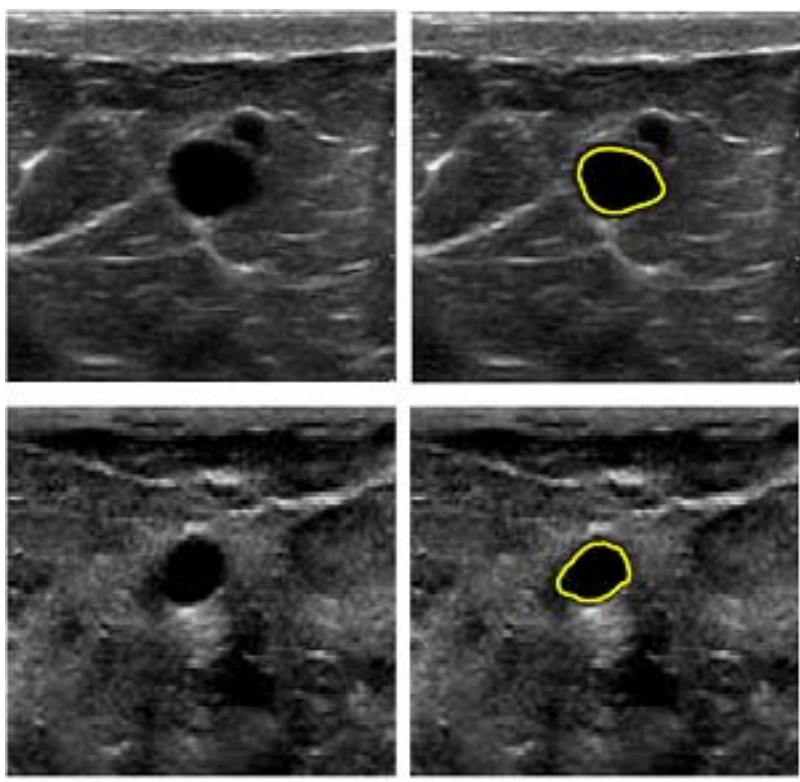

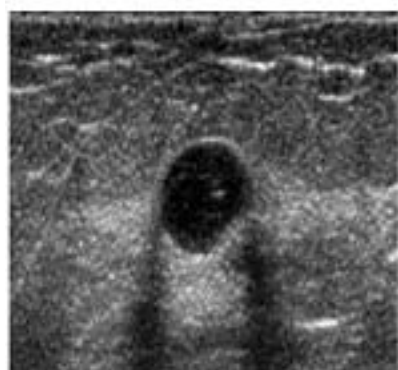

(a)

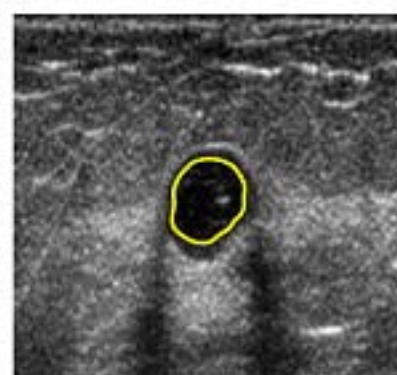

(b)
Figure 6. (a) medical ultrasound image with circular tissue's structure (b) Final result of segmentation after the proposed algorithm is applied.

leads to satisfactory results. Finally, it should be noted that the implementation of proposed algorithm on the images is time consuming, so these algorithm cannot be used for real-time image processing and this can be considered as a major disadvantage for proposed algorithm.

\section{CONCLUSION}

In this article, we try to apply the genetic algorithm to help solve some of the problems associated with the active contour and to use it for the segmentation of ultrasound images. Through the use of this algorithm, the problems of determining the contour's initial position and contour's entrapment within local minima no longer exist and the only thing needed for specifying the contours' initial positions is the center of the circles which could be found by determining the image's center of gravity. On the other hand, using this algorithm allows us to process only the region where the tissue is and to avoid processing the whole image. This reduces the time required for segmentation. The obtained results also show that this method of ultrasound image segmentation obtains acceptable accuracy.

\section{REFERENCES}

[1] Zhu, C.M., Gu, G.C., Liu, H.B., Shen, J. And Yu, H.L., (2008) Segmentation of ultrasound image based on cluster ensemble. IEEE International Symposium on Knowledge Acquisition and Modeling Workshop, Wuhan, 21-22 December 2008, 418-421. doi:10.1109/KAMW.2008.4810513

[2] Deka, B. and Ghosh, D. (2006) Watershed segmentation for medical ultrasound images. IEEE International Conference on Systems, Man, and Cybernetics, 6, 3186-3191. doi:10.1109/ICSMC.2006.384607

[3] Li, L., Fu, Y.X., Bai, P.R. and Mao, W.J. (2009) Medical ultrasound image segmentation based on improved watershed scheme. The 3rd International Conference on Bioinformatics and Biomedical Engineering, Beijing, 11-13 June 2009, 1-4.

[4] Li, L.H., Lin, J.L., Li, D.Y. and Wang, T.F. (2007) Segmentation of Medical Ultrasound Image Based on Markov Random Field. The 1st International Conference on Bioinformatics and Biomedical Engineering, Wuhan, 6-8 July 2007, 968-971.

[5] Sarti, A., Corsi, C., Mazzini, E. and Lamberti, C. (2005) Maximum likelihood segmentation of ultrasound images with rayleigh distribution. IEEE Transactions on Ultrasonic's, Ferroelectrics and Frequency Control, 52, 947-960. doi:10.1109/TUFFC.2005.1504017

[6] Michailovich, O. and Tannenbaum, A. (2007) Segmentation of medical ultrasound images using active contours. IEEE International Conference on Image Processing, 6, 513-516. doi:10.1109/ICIP.2007.4379878

[7] Kass, M., Witkin, A. and Terzopoulos, D. (1988) Snakes: active contour models. International Journal of Computer Vision, 1, 321-331. doi:10.1007/BF00133570

[8] Cohen, L.D. (1991) On active contour models and balloons. CVGIP: Image Understanding, 53, 211-218.

[9] Xu, C.Y. and Prince, J.L. (1998) Snakes, shapes, and gradient vector flow. IEEE Transactions on Image Processing, 7, 359-369.

[10] Cohen, L.D. and Cohen, I. (1993) Finite element methods for active contour models and balloons for 2-D and 3-D images. IEEE Transactions on Pattern Analysis and Machine Intelligence, 15, 1131-1147. doi:10.1109/34.244675

[11] L. Ballerini, (1993) Genetic snakes for medical images segmentation. In: Poli, R. Ed., Evolutionary image Analysis, Signal Processing and Telecommunications, Springer, London, 59-73.

[12] GholamAli Rezaei Rad, Mehdi Kashanian, (2006) Extraction of the breast cancer tumor in mammograms using genetic active contour. International Conference on Biomedical and Pharmaceutical Engineering, Singapore, 11-14 December 2006, 30-33.

[13] Sivanandam, S.N. and Deepa, S.N. (2008) Introduction to genetic algorithms. Springer-Verlag, New York.

[14] Gonzalez, R.C. and Woods, R.E. (2002) Digital image processing. 2nd Edition, Pearson Education, Inc., Upper Saddle River, New Jersey. 